\title{
Concurrent BRAF/MEK Inhibitors in BRAF V600-Mutant High-Grade Primary Brain Tumors
}

\author{
Karisa C. Schreck, MD, PhD; ; Andrew Guajardo, $\mathrm{MD}^{\mathrm{b}}$; Doris D.M. Lin, MD, PhD; \\ Charles G. Eberhart, MD, PhD ${ }^{\mathrm{b}}$; and Stuart A. Grossman, $\mathrm{MD}^{\mathrm{d}}$
}

\begin{abstract}
BRAF V600 mutations are being identified in patients with primary brain tumors more often as molecular testing becomes widely available. Targeted treatment with BRAF inhibitors has been attempted in individual cases with some responses, whereas others showed no response or developed resistance. Preclinical work suggests that gliomas could be more responsive to the concurrent use of BRAF and MEK inhibition for MAP kinase pathway suppression. This report presents 2 cases of malignant brain tumors with BRAF V600E mutations that were resistant to radiation and temozolomide, and reports on their response to targeted treatment with the BRAF and MEK inhibitors dabrafenib and trametinib. One patient with an anaplastic pleomorphic xanthoastrocytoma experienced a partial response for 14 months, demonstrated by progressive tumor shrinkage and clinical improvement; however, this was followed by clinical and radiographic progression. The patient with glioblastoma continued to have stable disease after 16 months of treatment. These cases are encouraging in a disease that urgently needs new treatments. Further work is necessary to understand response rates, duration, and survival in primary brain tumors.
\end{abstract}

J Natl Compr Canc Netw 2018;16(4):343-347 doi: 10.6004/jncen.2017.7052

As tumor profiling becomes more widespread, targetable mutations are being found in patients with brain tumors. One of these mutations, BRAF V600, allows this protein kinase to act as an oncogene activating the ERK signaling pathway, ${ }^{1}$ which leads to cell proliferation and survival. BRAF V600E is the most common mutation, although there are others that occur less frequently (V600K/D/R). BRAF-specific inhibitors have been developed and are effective in melanoma, with approximately $50 \%$ of metastatic melanomas showing response. ${ }^{2,3}$ Unfortunately, tumor resistance often emerges through several different mechanisms in an average of 5 to 7 months. ${ }^{3,4}$ Attempts have been made to bypass this resistance by blocking MEK, which is just downstream of BRAF, thereby making it more difficult for tumors to escape inhibition. However, if a BRAF inhibitor is fol-

\footnotetext{
aDepartment of Neurology, The Sidney Kimmel Comprehensive Cancer Center at Johns Hopkins; ${ }^{b}$ Department of Pathology, Johns Hopkins University; 'Department of Radiology and Radiological Science, Johns Hopkins Hospital; and ${ }^{d}$ The Sidney Kimmel Comprehensive Cancer Center at Johns Hopkins, Baltimore, Maryland.
}

Submitted July 5, 2017; accepted for publication October 18, 2017. lowed by a MEK inhibitor at progression, there is only marginal improvement in survival (1.8 months). ${ }^{5}$ More recently, the upfront combination of BRAF and MEK inhibitors has shown improved progression-free survival compared with monotherapy and is FDA-approved for advanced-stage melanoma.

BRAF V600 mutations have been discovered in a variety of adult and pediatric brain tumors, including pleomorphic xanthoastrocytomas (PXAs) (50\%-65\%), gangliogliomas (20\%-70\%), pilocytic astrocytomas (9\%$10 \%)$, low-grade gliomas (5\%-15\%), and adult glioblastomas (1\%-5\%)..$^{8-10}$ These mutations are less common in typical adult high-grade tumors, such as glioblastoma. However, they are relatively enriched in the epithelioid subtype of glioblastoma, ${ }^{10}$ and possibly in low-grade astrocytomas as well (5\%-15\%). ${ }^{11}$ There have been a few cas-

The authors have disclosed that they have no financial interests, arrangements, affiliations, or commercial interests with the manufacturers of any products discussed in this article or their competitors.

Correspondence: Karisa C. Schreck, MD, PhD, Department of Neurology, The Sidney Kimmel Comprehensive Cancer Center at Johns Hopkins, Suite 1M16, CRB II, 1550 Orleans Street, Baltimore, MD 21287.

Email: ksolt1@jhmi.edu 
Schreck et al

es in which targeted treatment with BRAF inhibitors has been successfully attempted, but resistance emerges in a few months, similar to what has been seen in melanoma and other tumor types. ${ }^{12-14}$ The emergence of resistance over time has been reproduced in preclinical glioma models, ${ }^{15,16}$ although these models have also shown that dual inhibition with BRAF and MEK inhibitors can prevent resistance.

This report presents 2 cases of treatment-refractory high-grade primary brain tumors treated with dual BRAF and MEK inhibitors: 1 anaplastic PXA and 1 epithelioid glioblastoma. Both cases showed clinical and radiographic response to treatment for $\geq 14$ months, highlighting the potential utility of BRAF-targeted therapy in high-grade astrocytomas refractory to standard treatment with radiation and temozolomide.

Approval was obtained from both patients to submit their clinical and imaging data for a case report, in agreement with the Johns Hopkins Institution Institutional Review Board requirements.

\section{Case Reports}

\section{Case 1}

A 16-year-old man presented with focal seizures and a left temporal lobe mass in 1998, which was observed until 2002 when he underwent surgery, and pathology results showed PXA. He underwent a second surgery in 2004 after demonstrating progression, with pathology still showing typical microscopic features of PXA, WHO grade II (Figure 1A), and then experienced stable disease for the next 10 years. In 2014, at age 32 years, radiographic and clinical progression prompted his third surgery. Microscopic examination revealed an increased number of mitotic figures, resulting in a diagnosis of anaplastic PXA (Figure 1B). Molecular studies showed a BRAF V600E mutation and no IDH1 mutation by immunohistochemistry. His postoperative course was complicated by infection and placement of a ventriculoperitoneal shunt. He was treated with radiation and concurrent temozolomide, followed by 6 cycles of adjuvant temozolomide. He experienced progression 4 months later and received 1 cycle of salvage BCNU with no signs of tumor response. In October 2015, he underwent another resection of his growing tumor for presumed progression, with pathology showing persistent anaplastic PXA morphology with
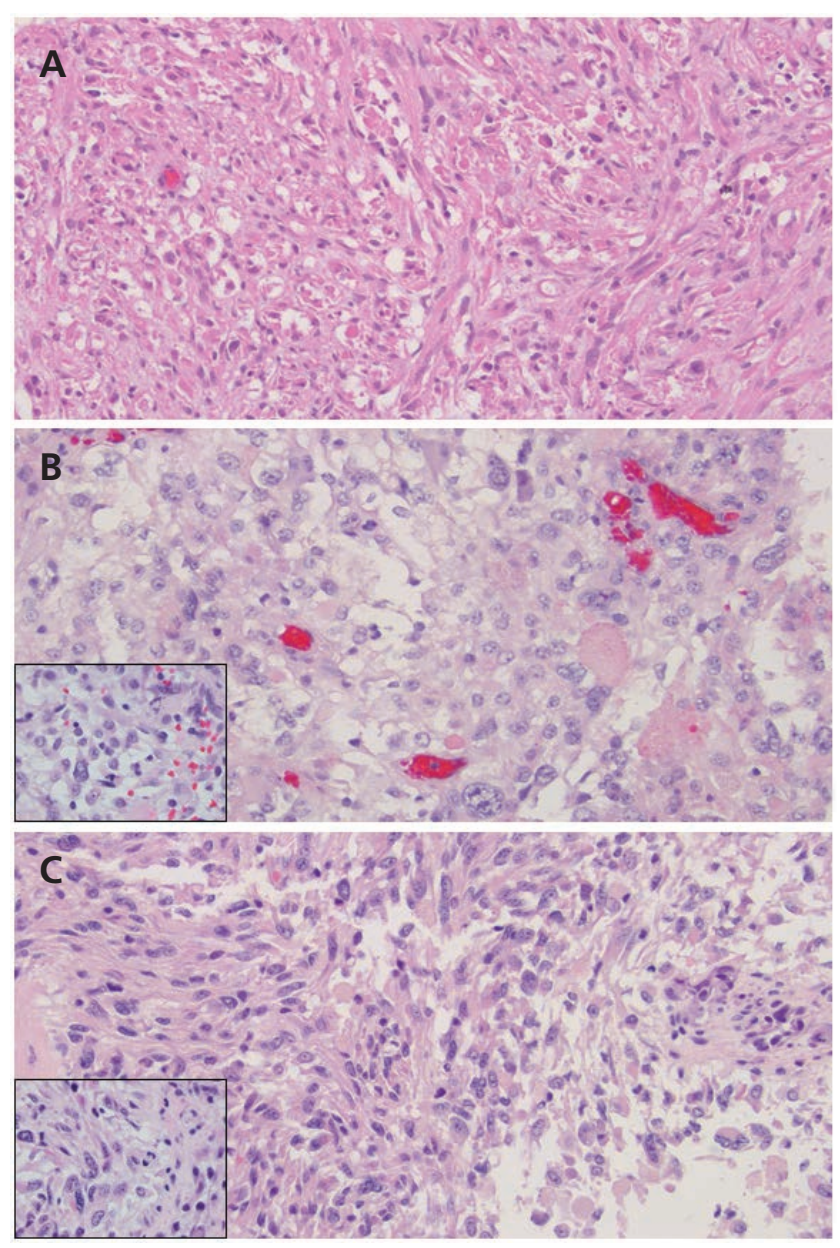

Figure 1. Histologic features of case 1 (hematoxylin-eosin, original magnification $\times 20$ ). (A) The 2004 resection shows a moderately cellular neoplasm with somewhat spindled architecture. Nuclear pleomorphism, dense eosinophilic cytoplasm, occasional multinucleated cells, and frequent eosinophilic granular bodies (EGBs) were all present, but mitotic activity was inconspicuous. (B) The recurrence in 2014 had elevated mitotic activity (inset) with $>5$ mitoses per high-power field, consistent with anaplastic pleomorphic xanthoastrocytoma (PXA), WHO grade III. (C) In 2015, the recurrence still showed classic PXA features, including scattered bizarre nuclei with prominent atypia and EGBs, but mitotic activity remained elevated (inset).

variable pleomorphism, eosinophilic granular bodies, and frequent mitoses (Figure 1C). In the setting of progression, he began receiving repeat irradiation. However, this was discontinued abruptly after only 4 of 20 planned treatments when he experienced an acute left internal capsule stroke. In January 2016, he began therapy with dabrafenib at $150 \mathrm{mg}$ twice daily and trametinib at $2 \mathrm{mg}$ daily, with a Karnofsky performance status (KPS) of 50 and an ECOG performance status (PS) of 3.

Treatment was complicated by fevers and a rash that responded to steroids. The steroids were titrated to symptoms, with a maximum dosage of $4 \mathrm{mg} / \mathrm{d}$, and 
were stopped 8 months after treatment initiation. His clinical condition and MRI scans steadily improved, and he became functionally independent. In February 2017, he presented to the hospital leaking cerebrospinal fluid through his nose and was admitted with pneumocephalus. This was the result of tumor regression, which had previously eroded through the subarachnoid space and bone. He was treated surgically with an autologous fascial graft to the skull base and a mesh cranioplasty. A small amount of residual tumor was harvested at that time and pathology was notable for scattered cells consistent with PXA and inconspicuous mitotic activity. The tissue continued to show the BRAF V600E mutation on molecular testing.

He continued to do well on BRAF/MEK inhibitors, with a KPS of 70, for 14 months after initiating therapy. He also continued to show steadily decreasing volume of enhancing tumor on MRI over the course of treatment (Figure 2A, B). Tumor volumes were quantified using the lesion segmentation function in the CARESTREAM PACS system (Carestream Health, Inc.). Unfortunately, his routine MRI scan performed 16 months after treatment initiation showed signs of tumor progression and BRAF/MEK inhibition was discontinued. Detailed molecular testing on his tissue at that time showed the BRAF V600E mutation continued to be expressed and that there were no additional mutations in BRAF. Additionally, there were missense muta- tions of unclear significance in FLT4, NOTCH1, and TSC2, but no known resistance mechanisms.

\section{Case 2}

A 23-year-old woman presented in November 2008 with hemiplegia, diplopia, and sensory changes and was diagnosed with left frontal epithelioid glioblastoma. The tumor was glial fibrillary acidic proteinpositive, but IDH, MGMT, and BRAF testing were not performed at that time. She underwent a gross total resection followed by radiation with concurrent temozolomide. One month later, she received an additional stereotactic boost of 25 Gy and adjuvant temozolomide for 12 cycles. In September 2010, she underwent surgery for suspected disease progression based on imaging, but pathology showed gliosis. In November 2014, she had a new local nodule resected and 2.5 Gliadel wafers (Eisai Inc., Woodcliff, NJ) were placed. Histopathology of the recurrent epithelioid glioblastoma demonstrated abundant eosinophilic cytoplasm, frequent mitotic activity, vascular proliferation, and necrosis. Molecular testing at that time revealed a BRAF V600E mutation and no IDH1/2 mutations. She was observed until there was evidence of radiographic tumor progression on 2 serial MRI scans 1 month apart. In January 2016, she was started on dabrafenib at $150 \mathrm{mg}$ twice daily and trametinib at 2 mg daily; her KPS was 70 and ECOG PS was 2.

In the first few weeks of starting treatment, she experienced fevers, hypotension, and meningismus,
A

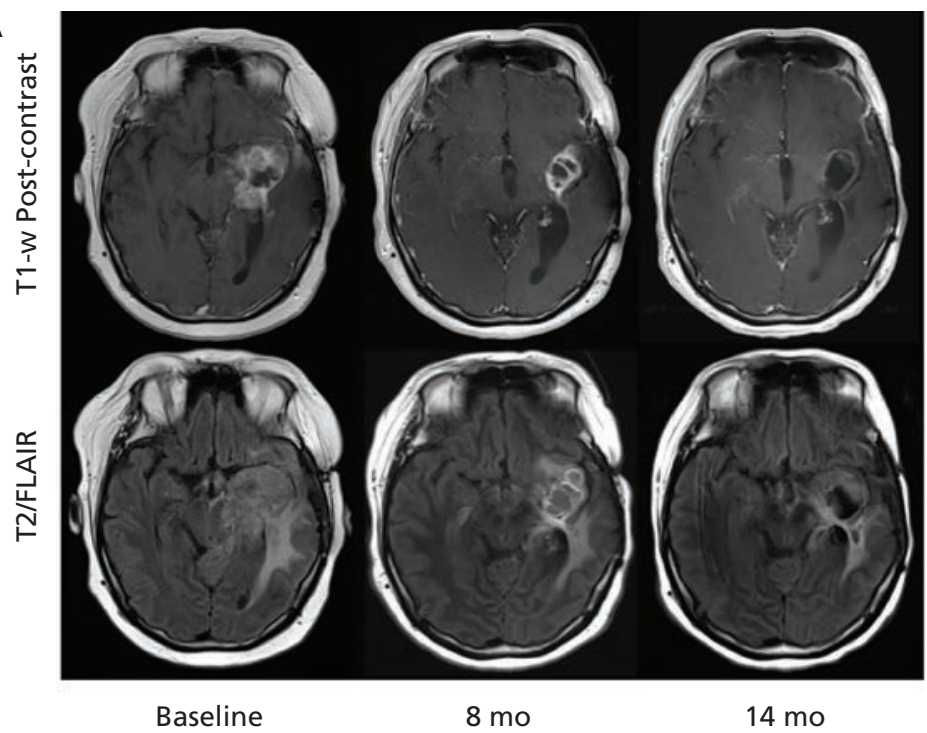

B

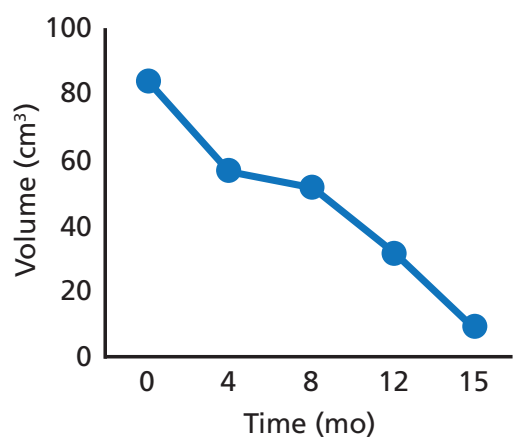

Figure 2. Case 1 imaging response to BRAF-directed therapy (A) T2-flair and T1-weighted postgadolinium MR images showing progressive reduction of enhancing tumor size in the left temporal lobe with (B) volumetric measurements plotted against time. 


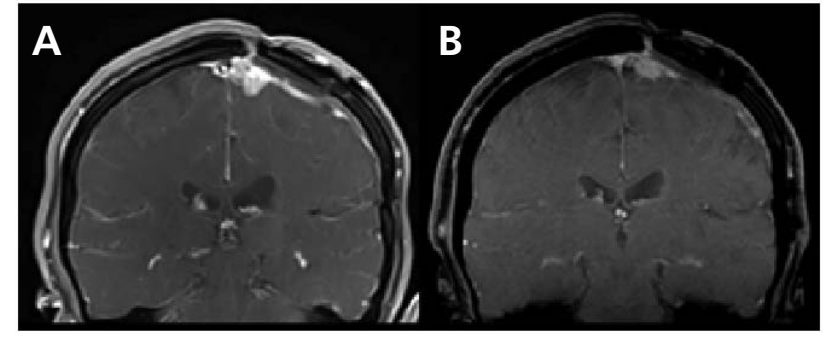

Figure 3. Case 2 imaging response to BRAF-directed therapy. CT1weighted postgadolinium MR images from $(A)$ before treatment and (B) after 14 months of treatment showing reduced bulk of enhancing tumor in the left parasagittal frontal region.

causing her to stop the medications. She restarted dabrafenib at a reduce dose of $100 \mathrm{mg}$ twice daily, trametinib at $2 \mathrm{mg}$ daily, and dexamethasone at $2 \mathrm{mg}$ daily. She tolerated this regimen well, but became symptomatic if dexamethasone was decreased. At the time of writing, she has completed 16 months of therapy and has shown stable disease on MRI per RANO criteria $^{17}$ (Figure 3 ) and has stable functional status. She continues on $2 \mathrm{mg}$ daily of dexamethasone.

\section{Discussion}

This manuscript reports on 2 people with BRAF V600-mutated primary brain tumors treated as adults with concurrent BRAF and MEK inhibitors. In both of these cases, patients received $\geq 1$ rounds of standard chemotherapy and radiation, and experienced disease progression before initiating treat- ment with BRAF and MEK inhibitors. In response to treatment with BRAF/MEK inhibitors, the patients had either stable disease or partial response for $\geq 14$ months. This is unusual in high-grade primary brain tumors, which are relentlessly progressive once they begin to grow and do not generally undergo spontaneous regression or senescence. It is also notable because standard-of-care second- or third-line treatments have limited efficacy.

It is interesting that the first case showed progressive tumor shrinkage over the course of 14 months. This is different from what is typically seen in melanoma, even brain metastases, where most of the response occurs in the first few weeks of treatment. ${ }^{18}$ This observation suggests potentially different pharmacokinetics in this tumor type (PXA), or a differential dependence on the ERK signaling pathway compared with other tumor types. Alternatively, it may be due to less drug accumulation within the tumor and brain parenchyma, because preclinical models suggest both dabrafenib and trametinib have limited central nervous system penetration due to active drug efflux..$^{19,20}$ This could potentially result in a slow response over time as tumor areas are gradually exposed to drug.

It is unclear whether BRAF V600-mutated primary brain tumors respond differentially to BRAF or MEK monotherapy versus a combination. Preclinical work has shown that BRAF inhibitors transiently decrease ERK signaling in glioma cell lines. ${ }^{21}$ Coinhibition with BRAF and MEK inhibitors pro-

\begin{tabular}{|c|c|c|c|c|c|c|c|}
\hline & Age, $y /$ Sex & Histology & Prior Rx & BRAF $\mathrm{Rx}$ & Time on $\mathbf{R x}$ & Best Outcome & Reference \\
\hline 1 & $34 / F$ & PXA & $\mathrm{RT}, \mathrm{TMZ}, \mathrm{PCV}$ & Vemurafenib & $4 \mathrm{mo}$ & SD & Chamberlain $^{12}$ \\
\hline 2 & $47 / F$ & PXA & RT, TMZ, PCV & Vemurafenib & $10 \mathrm{mo}$ & $P R$ & Chamberlain $^{12}$ \\
\hline 3 & $53 / \mathrm{M}$ & PXA & $\mathrm{RT}+\mathrm{TMZ}, \mathrm{PCV}$ & Vemurafenib & $10 \mathrm{mo}$ & PR & Chamberlain ${ }^{12}$ \\
\hline 4 & 43/M & Anaplastic PXA & $\mathrm{RT}+\mathrm{TMZ}, \mathrm{PCV}$ & Vemurafenib & $6 \mathrm{mo}$ & Progression & Chamberlain $^{12}$ \\
\hline 5 & $26 / \mathrm{M}$ & Epithelioid GBM & None & Vemurafenib & $1 \mathrm{wk}$ & PR & Leaver et $\mathrm{al}^{14}$ \\
\hline 6 & 39/M & Anaplastic PXA & RT & Vemurafenib & $2 \mathrm{mo}$ & CR & Leaver et al ${ }^{14}$ \\
\hline 7 & $41 / \mathrm{M}$ & Anaplastic PXA & $\mathrm{TMZ}, \mathrm{RT}+\mathrm{TMZ}$ & Vemurafenib & $3 \mathrm{mo}$ & PR & Lee et $\mathrm{al}^{22}$ \\
\hline 8 & $35 / F$ & PXA & None & Dabrafenib & $3 \mathrm{mo}$ & PR & Usubalieva et $\mathrm{al}^{23}$ \\
\hline 9 & $25 / M$ & $\begin{array}{l}\text { HGG from } \\
\text { ganglioglioma }\end{array}$ & $\begin{array}{l}\text { RT+TMZ with adjuvant TMZ } \\
\times 4\end{array}$ & $\begin{array}{l}\text { Dabrafenib, Optune } \\
\text { (Novocure) }\end{array}$ & $\geq 24 \mathrm{mo}$ & CR & Meletath et $a^{24}$ \\
\hline 10 & $21 / F$ & Anaplastic PXA & $R T$, vemurafenib & Dabrafenib & $\geq 16 \mathrm{mo}$ & CR & Brown et $\mathrm{al}^{25}$ \\
\hline 11 & $35 / \mathrm{M}$ & Anaplastic PXA & $\mathrm{RT}+\mathrm{TMZ}, \mathrm{BCNU}$ & Dabrafenib, trametinib & $\geq 14 \mathrm{mo}$ & PR & Present study \\
\hline 12 & $32 / \mathrm{F}$ & Epithelioid GBM & RT+TMZ, Gliadel & Dabrafenib, trametinib & $\geq 14 \mathrm{mo}$ & PR & Present study \\
\hline
\end{tabular}

Abbreviations: $\mathrm{CR}$, complete response; F, female; GBM, glioblastoma; HGG, high-grade glioma; $\mathrm{M}$, male; PCV, procarbazine/lomustine/vincristine; PR, partial response; PXA, pleomorphic xanthoastrocytoma; RT, radiation therapy; Rx, treatment; SD, stable disease; TMZ, temozolomide. 
BRAF/MEK Inhibitors in Brain Tumors

vides a more durable response in vitro and in vivo. ${ }^{16}$ However, to date, case reports in adults with primary brain tumors have only examined BRAF inhibition, resulting in a mix of response and progression (Table 1). ${ }^{12,14,22-25}$ Whether adding an MEK inhibitor would change the clinical course is unknown. To answer this question, 2 clinical trials evaluating the activity of dabrafenib and trametinib in pediatric patients with recurrent BRAF V600-mutated low- or high-grade gliomas (ClinicalTrials.gov identifiers: NCT02124772 and NCT02684058, respectively) are open or planned to open shortly.

Notably, the effect of a BRAF V600 mutation on the natural history of brain tumors in adults remains to be determined. One retrospective review of young adults aged 17 to 35 years with glioblastomas found a more favorable overall survival among those with BRAF mutations than those without. ${ }^{26}$ Another retrospective review of PXAs also found a more favorable overall survival associated with BRAF V600 mutation than without; however, this could not be controlled for tumor grade because more of the mutations were seen in grade II than grade III PXA. ${ }^{27}$ Regardless, in the cases reported herein, the tumors were progressive despite aggressive treatment with radiation and chemotherapy, necessitating a new treatment strategy. Dual BRAF/MEK inhibition precipitated tumor response or stable disease for more than a year, making this an interesting potential treatment for other patients with similar tumors.

\section{Conclusions}

The cases described in this report demonstrate that targeting the BRAF V600 mutation with dual BRAF/ MEK inhibition in recurrent primary brain tumors may produce a durable response. Further studies are needed to determine formal response rates, response durations, the impact of this therapy on survival, and the entry of these agents into brain parenchyma.

\section{References}

1. Davies H, Bignell GR, Cox C, et al. Mutations of the BRAF gene in human cancer. Nature 2002;417:949-954.

2. Chapman $P B$, Hauschild $A$, Robert $C$, et al. Improved survival with vemurafenib in melanoma with BRAF V600E mutation. N Engl J Med 2011;364:2507-2516.

3. Hauschild A, Grob JJ, Demidov LV, et al. Dabrafenib in BRAF-mutated metastatic melanoma: a multicentre, open-label, phase 3 randomised controlled trial. Lancet 2012;380:358-365.

4. Sosman JA, Kim KB, Schuchter L, et al. Survival in BRAF V600mutant advanced melanoma treated with vemurafenib. N Engl J Med 2012;366:707-714.

5. Kim KB, Kefford R, Pavlick AC, et al. Phase II study of the MEK1/MEK2 inhibitor trametinib in patients with metastatic BRAF-mutant cutaneous melanoma previously treated with or without a BRAF inhibitor. J Clin Oncol 2013;31:482-489.

6. Robert C, Karaszewska B, Schachter J, et al. Improved overall survival in melanoma with combined dabrafenib and trametinib. N Engl J Med 2015;372:30-39.

7. Long GV, Stroyakovskiy D, Gogas H, et al. Dabrafenib and trametinib versus dabrafenib and placebo for Val600 BRAF-mutant melanoma: a multicentre, double-blind, phase 3 randomised controlled trial. Lancet 2015;386:444-451.

8. Schindler G, Capper D, Meyer J, et al. Analysis of BRAF V600E mutation in 1,320 nervous system tumors reveals high mutation frequencies in pleomorphic xanthoastrocytoma, ganglioglioma and extra-cerebellar pilocytic astrocytoma. Acta Neuropathol 2011;121:397-405.

9. Dahiya S, Emnett RJ, Haydon DH, et al. BRAF-V600E mutation in pediatric and adult glioblastoma. Neuro Oncol 2014;16:318-319.

10. Behling F, Barrantes-Freer A, Skardelly M, et al. Frequency of BRAF V600E mutations in 969 central nervous system neoplasms. Diagn Pathol 2016;11:55

11. Chi AS, Batchelor TT, Yang D, et al. BRAF V600E mutation identifies a subset of low-grade diffusely infiltrating gliomas in adults. J Clin Oncol 2013;31:e233-236.

12. Chamberlain MC. Salvage therapy with BRAF inhibitors for recurrent pleomorphic xanthoastrocytoma: a retrospective case series. J Neurooncol 2013;114:237-240.

13. Hofer S, Berthod G, Riklin C, et al. BRAF V600E mutation: a treatable driver mutation in pleomorphic xanthoastrocytoma (PXA). Acta Oncol 2016;55:122-123.

14. Leaver KM, Zhang N, Ziskin JL, et al. Response of metastatic glioma to vemurafenib. Neurooncol Pract 2016;3:268-271.

15. Grossauer S, Koeck K, Murphy NE, et al. Concurrent MEK targeted therapy prevents MAPK pathway reactivation during BRAFV600E targeted inhibition in a novel syngeneic murine glioma model. Oncotarget 2016;7:75839-75853.

16. Zhang J, Yao TW, Hashizume R, et al. Combined BRAFV600E and MEK blockade for BRAFV600E-mutant gliomas. J Neurooncol 2016;131:495505.

17. Wen PY, Macdonald DR, Reardon DA, et al. Updated response assessment criteria for high-grade gliomas: response assessment in neuro-oncology working group. J Clin Oncol 2010;28:1963-1972.

18. Falchook GS, Long GV, Kurzrock R, et al. Dabrafenib in patients with melanoma, untreated brain metastases, and other solid tumours: a phase 1 dose-escalation trial. Lancet 2012;379:1893-1901.

19. Mittapalli RK, Vaidhyanathan S, Dudek AZ, Elmquist WF. Mechanisms limiting distribution of the threonine-protein kinase B-RaF(V600E) inhibitor dabrafenib to the brain: implications for the treatment of melanoma brain metastases. J Pharmacol Exp Ther 2013;344:655-664.

20. Vaidhyanathan S, Mittapalli RK, Sarkaria JN, Elmquist WF. Factors influencing the CNS distribution of a novel MEK-1/2 inhibitor: implications for combination therapy for melanoma brain metastases. Drug Metab Dispos 2014;42:1292-1300.

21. Nicolaides TP, Li H, Solomon DA, et al. Targeted therapy for BRAFV600E malignant astrocytoma. Clin Cancer Res 2011;17:7595-7604.

22. Lee EQ, Ruland $S$, LeBoeuf NR, et al. Successful treatment of a progressive BRAF V600E-mutated anaplastic pleomorphic xanthoastrocytoma with vemurafenib monotherapy. J Clin Oncol 2016;34:e87-89.

23. Usabalieva A, Pierson CR, Kavran CA, et al. Primary meningeal xanthoastrocytoma with anaplastic features: a report of 2 cases, one with BRAF (V600E) mutation and clinical response to the BRAF inhibitor dabrafenib. J Neuropathol Exp Neurol 2015;74:960-969.

24. Meletath SK, Pavlick D, Brennan $T$, et al. Personalized treatment for a patient with a BRAF V600E mutation using dabrafenib and a tumor treatment fields device in a high-grade glioma arising from ganglioglioma. J Natl Compr Canc Netw 2016;14:1345-1350.

25. Brown NF, Carter T, Mulholland P. Dabrafenib in BRAFV600E-mutated anaplastic pleomorphic xanthoastrocytoma. CNS Oncol 2017;6:5-9.

26. Zhang RQ, Shi Z, Chen $H$, et al. Biomarker-based prognostic stratification of young adult glioblastoma. Oncotarget 2016;7:5030-5041.

27. Ida CM, Rodriguez FJ, Burger PC, et al. Pleomorphic xanthoastrocytoma: natural history and long-term follow-up. Brain Pathol 2015;25:575-586. 